\title{
Feohifomicosis causada por Colletotrichum gloeosporioides y Alternaria infectoria en un paciente trasplantado renal
}

\author{
Marilia Ogawa, Viviane Reis, Patricio Godoy, Fernando Gatti de Menezes, Milvia Enokihara y Jane Tomimori
}

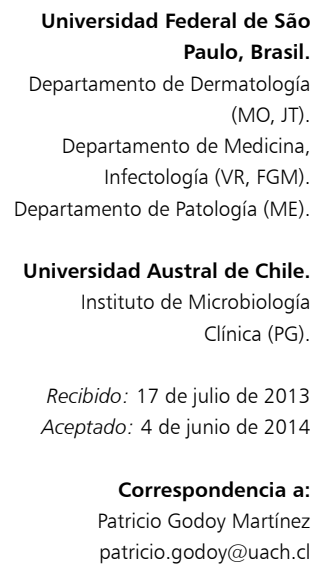

Universidad Federal de São Paulo, Brasil. Departamento de Dermatología (MO, JT)

Departamento de Medicina, Infectología (VR, FGM) Departamento de Patología (ME)

Universidad Austral de Chile. Instituto de Microbiología Clínica (PG).

Recibido: 17 de julio de 2013 Aceptado: 4 de junio de 2014

Correspondencia a: Patricio Godoy Martínez patricio.godoy@uach.cl

\section{Phaeohyphomycosis caused by Colletotrichum gloeosporioides and Alternaria infectoria in renal transplant recipient}

Several species of black fungi have been reported as agents of subcutaneous phaeohyphomycosis. Although most of these fungi are considered opportunistic pathogens, they play an important role in phaeohyphomycosis, a disease considered an emergent mycosis among solid organ recipients. We report a case of phaeohyphomycosis caused by Alternaria infectoria of the left hand and the $4^{\text {th }}$ finger of the right hand of a 68-year-old male who underwent a renal transplant 35 months before. The lesion was treated with surgical excision. One year later, the patient presented a new lesion on the $5^{\text {th }}$ finger of the right hand, but this time caused by Colletotrichum gloeosporioides that was also removed surgically. Both lesions did not relapse after being removed. Antifungal susceptibility testing was performed against five antifungal drugs (amphotericin B, itraconazole, flucytosine, fluconazole and voriconazole). Alternaria infectoria was resistant to all five drugs and C. gloeosporioides was sensitive only to amphotericin B and voriconazole. We emphasize the need of histopathologic and microbiologic studies of new lesions of phaeohyphomycosis, since in this case the same patient was infected twice by two different fungi.

Key words: Phaeohyphomycosis, renal transplant, Alternaria infectoria, Colletotrichum gloeosporioides.

Palabras clave: Feohifomicosis, trasplante renal, Alternaria infectoria, Colletotrichum gloeosporioides.

\section{Introducción}

$\mathrm{E}$ 1 uso de fármacos inmunosupresores en los pacientes sometidos a trasplantes de órganos sólidos aumenta la susceptibilidad a enfermedades infecciosas, causa importante de morbi-mortalidad en trasplantados. Las micosis superficiales y subcutáneas son frecuentes en pacientes en fase de mantención con tratamiento inmunosupresor. La feohifomicosis es una micosis emergente que se ha observado en pacientes trasplantados de órganos sólidos, causada por una amplia variedad de especies fúngicas, la mayoría de estos considerados patógenos oportunistas ${ }^{1,2,3,4}$.

Más de 50 géneros de hongos que presentan melanina y considerados saprobios en la naturaleza han sido reconocidos como agentes de feohifomicosis. Causan una gran variedad de manifestaciones clínicas destacando las micosis cutáneas, subcutáneas y profundas. La feohifomicosis es una infección oportunista y la exposición del paciente a materiales contaminados presentes en la naturaleza (maderas y plantas) pueden ser un factor de riesgo importante. La enfermedad invasora, aunque no es común, ocurre con frecuencia en pacientes inmunocomprometidos, debido principalmente a deficiencia de la inmunidad celular ${ }^{3}$.

Las especies del género Alternaria spp. son hongos que presentan melanina y que incluyen cerca de 80 especies y variedades. Los criterios taxonómicos del género, basados en la morfología del conidio, fueron establecidos por Withshire (1933). De las especies del género, ocho han sido descritas como agentes de cuadros clínicos: A. alternata, $A$. brassicicola, A. infectoria, A. chartarum, A. stemphylioides, A. dianthicola, A. pluriseptata y A. tenuissima ${ }^{5,6}$. Hongos pertenecientes al género Alternaria son cosmopolitas, siendo encontrados en diferentes climas y temperaturas. Muchas especies son consideradas saprobias del suelo y aire y han sido descritas como importantes fitopatógenos. También han sido aislados como microbiota transitoria de piel y conjuntiva de seres humanos y otros animales ${ }^{7,8}$.

El género Colletotrichum es considerado un importante fitopatógeno, clasificado dentro de los Coelomycetous, en el orden Melanconiales. Es de distribución mundial pero se encuentra principalmente en regiones subtropicales y tropicales. Las especies de Colletotrichum causan pérdidas económicas importantes en la agricultura por producir un cuadro denominado antracnosis en plantas ${ }^{9}$. Cinco especies de Colletotrichum se han vinculado como agente causal de infecciones en seres humanos: $C$. coccodes, C. crassipes, C. dematium, $C$. gloeosporioides y $C$. graminicola $^{7,10,11}$. En Chile, no existen reportes de casos humanos producidos por este hongo, pero se ha descrito en cultivos de quinoa en la Región de la Araucanía.

Presentamos el caso de un paciente trasplantado renal 
que desarrolló dos nódulos en su mano izquierda causado por Alternaria infectoria y, un año más tarde presentó un nódulo en su mano derecha causado por Colletotrichum gloeosporioides.

\section{Caso clínico}

Paciente de nacionalidad brasileña de sexo masculino, de 68 años de edad, consultó en Servicio de Dermatología del Hospital de Sao Paulo por un nódulo de color púrpura, de 2-3 cm de diámetro, en el dorso de la mano izquierda (Figura 1) y una lesión papular, de $0,3 \mathrm{~cm}$ de diámetro, en la $4^{\circ}$ falange de la mano derecha (Figura 2), ambas de tres meses de evolución. Las lesiones eran indoloras y de consistencia fibro-elástica. No refirió otros síntomas. Como antecedente, el paciente había sido sometido a un trasplante renal 35 meses antes de la aparición de las lesiones y se encontraba con tratamiento inmunosupresor continuo con tacrolimus ( $7 \mathrm{mg} /$ día), azatioprina $(50 \mathrm{mg} /$ día) y prednisona $(10 \mathrm{mg} /$ día $)$. El paciente no refirió ningún trauma, sin embargo, su profesión era jardinero por lo que pudo haber adquirido esta micosis de implantación por su trabajo con plantas. Ambas lesiones fueron removidas quirúrgicamente sin otro tratamiento. El material fue enviado a exámenes histopatológicos y micológicos al Laboratorio Especial de Micología (LEMI) de la Universidad Federal de Sao Paulo. Las tinciones con hematoxilina-eosina, Gomori y Fontana-Masson, revelaron la presencia de hifas dematiáceas en ambas lesiones y posteriormente fue identificado como $A$. infectoria.

Un año después, el paciente presentó otro nódulo, esta vez en la $5^{\circ}$ falange de la mano derecha (Figura 3 ). Se realizó biopsia y cultivo de la lesión, aislándose $C$. gloeosporioides. El paciente se encontraba con una dosis menor de tacrolimus ( $3 \mathrm{mg} /$ día), pero con azatioprina y prednisona en las mismas dosis. El paciente negó haber tenido algún trauma local, pero nuevamente relató que trabajaba frecuentemente en su jardín. Se realizó escisión quirúrgica completa de la lesión, sin tratamiento antifúngico. Después de un año de control no se observaron nuevas lesiones.

\section{Estudio micológico}

\section{Alternaria infectoria}

Las biopsias fueron cultivadas en agar Sabouraud (SDA) y agar papa (PDA), donde se observaron colonias de tonalidad gris a oliva, de aspecto aterciopelado (Figura 4). La micromorfología demostró la presencia de conidióforos flexuosos, de color café oscuros, no ramificados, con conidios ovoides, rugosos, con el septo transversal $\mathrm{u}$ oblicuo y a menudo con una extensión apical muy corta, la que da origen a un conidióforo secundario (Figura 5). El agente fue identificado como $A$. infectoria.

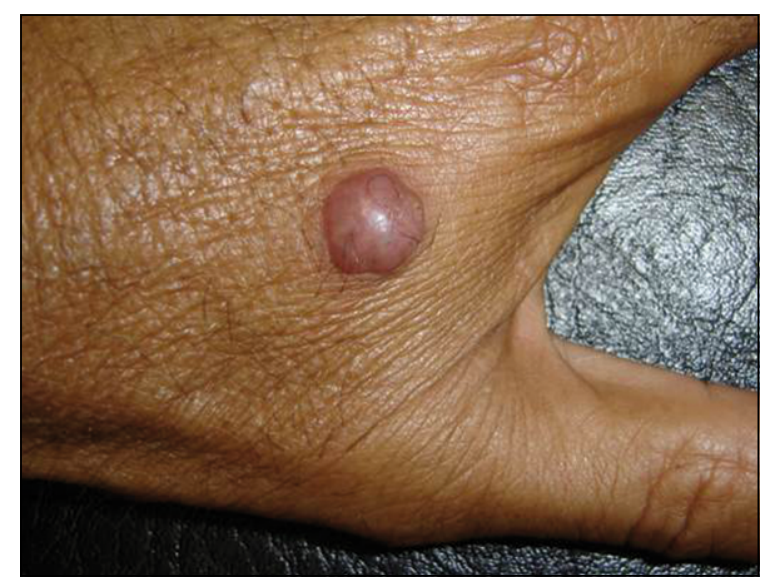

Figura 1. Nódulo púrpura en el dorso de la mano izquierda.

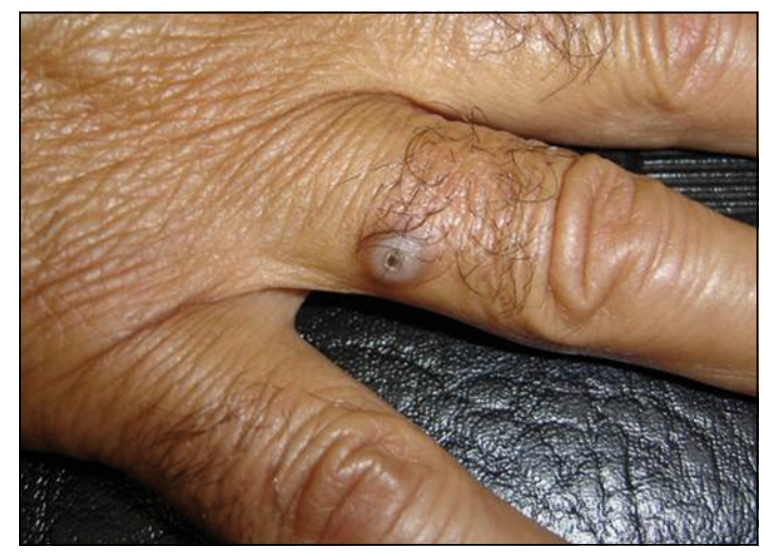

Figura 2. Lesión tipo pápula en la $4^{\circ}$ falange de la mano derecha.

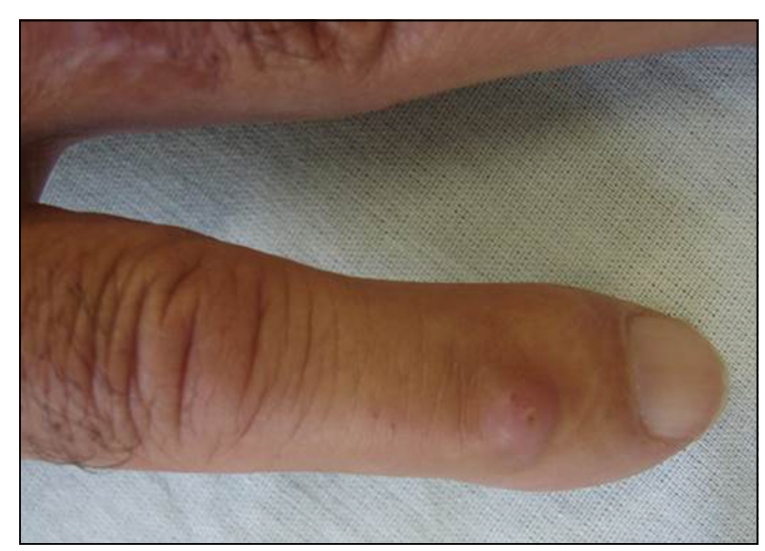

Figura 3. Nódulo violáceo en la $5^{\circ}$ falange de la mano derecha.

\section{Colletotrichum gloeosporioides}

Las colonias fúngicas obtenidas del material de la biopsia y del aspirado del nódulo fueron inoculadas en agar papa dextrosa (PDA) e incubadas a temperatura ambiente. Las colonias crecieron rápidamente, presentando una tonalidad gris a café, con manchas rosáceas. Al reverso, 


\section{Caso Clínico}

Figura 4. Características de colonia de Alternaria infectoria en agar papa (PDA), donde se observa un micelio aterciopelado de tonalidad gris a negra.
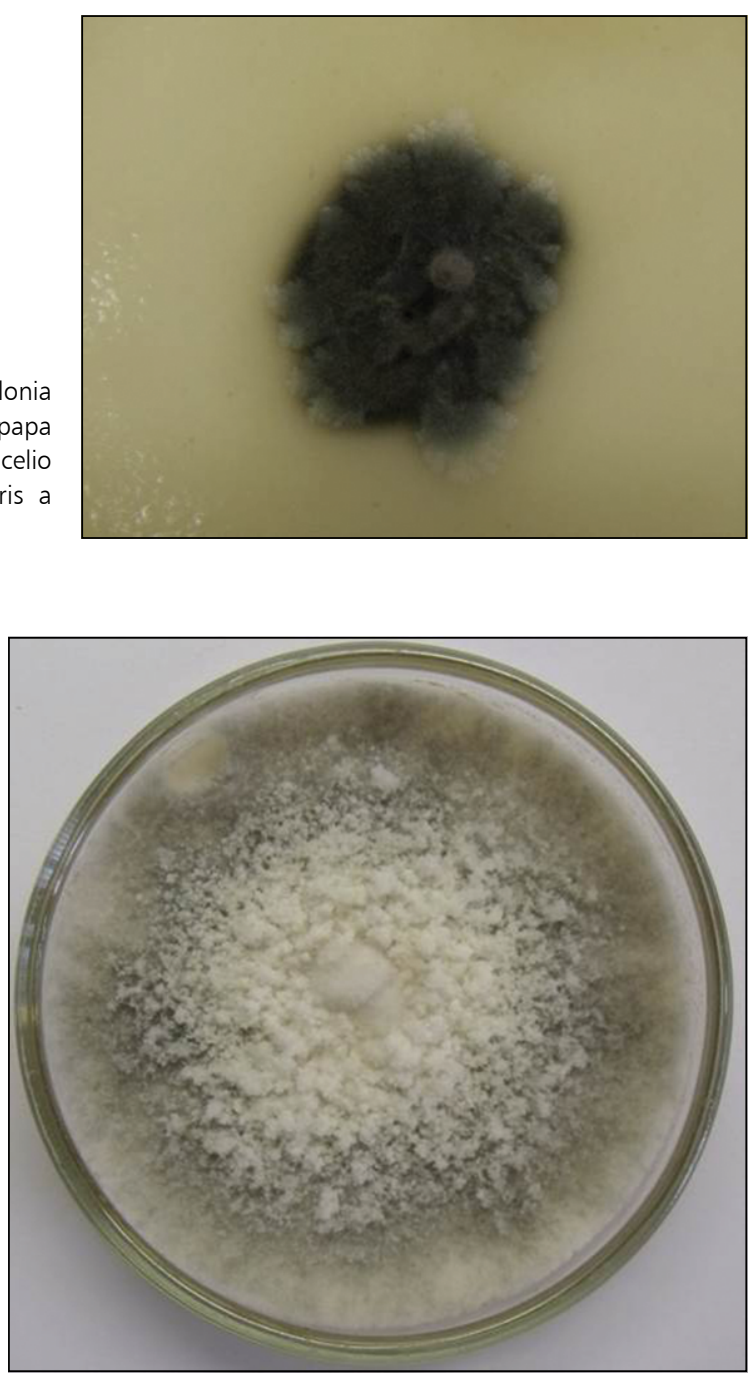

Figura 6. Características de colonia de Colletotrichum gloeosporioides en agar papa (PDA), donde se observa un micelio algodonoso de tonalidad gris a café con algunas colonias de color rosado.

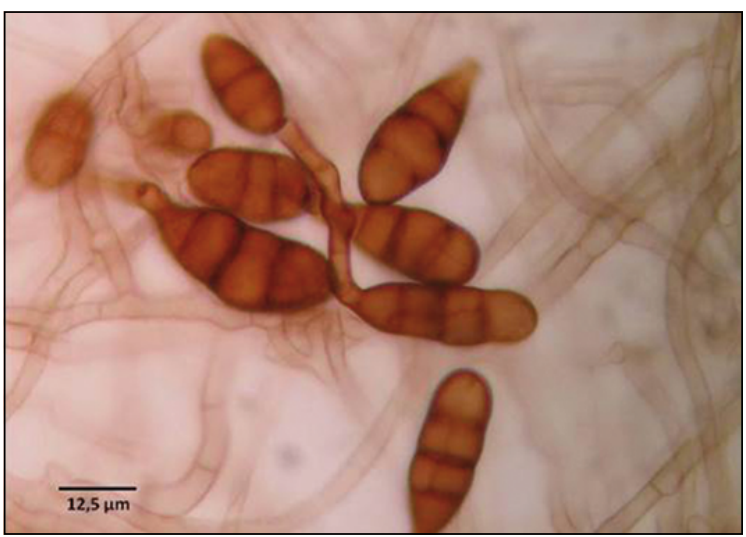

Figura 5. Características de la micromorfología de Alternaria infectoria, utilizando tinción de lactofenol de Amann se observan conidióforos flexuosos con conidios ovoides, rugosos, con el septo transversal u oblicuo (400x).

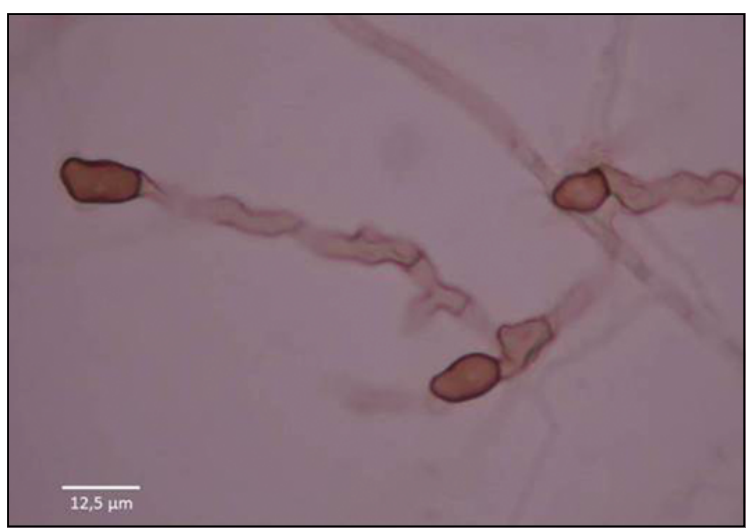

Figura 7. Características de la micromorfología utilizando tinción de lactofenol de Amann se observan células apresorias características de C. gloeosporioides (400x).

era de color pardo (Figura 6). En la micromorfología se observaron conidios rectos, cilíndricos, hialinos y obtusos en el ápice. Además numerosas células denominadas apresorias de aspecto claviforme, triangular o irregular con pigmento oscuro (Figura 7). El hongo fue identificado como C. gloeosporioides.

Tabla 1. Susceptibilidad antifúngica de Colletotrichum gloeosporioides y Alternaria infectoria

\begin{tabular}{|lccccc|} 
Cepas & $\begin{array}{c}\text { Anfotericina B } \\
\boldsymbol{\mu} \mathbf{g} / \mathbf{m L}\end{array}$ & $\begin{array}{c}\text { Itraconazol } \\
\boldsymbol{\mu} \mathbf{g} / \mathbf{m L}\end{array}$ & $\begin{array}{c}\text { Fluorcitosina } \\
\boldsymbol{\mu} \mathbf{g} / \mathbf{m L}\end{array}$ & $\begin{array}{c}\text { Fluconazol } \\
\boldsymbol{\mu} \mathbf{g} / \mathbf{m L}\end{array}$ & $\begin{array}{c}\text { Voriconazol } \\
\boldsymbol{\mu} \mathbf{g} / \mathbf{m L}\end{array}$ \\
C. gloeosporioides & 0,5 & 2 & $>64$ & $>64$ & 0,5 \\
A. infectoria & 1 & 16 & $>64$ & $>64$ & 8,0 \\
\hline
\end{tabular}

\section{Prueba de susceptibilidad antifúngica}

A pesar de que no están establecidos los puntos de corte para la definición de susceptibilidad antifúngica contra hongos melanizados, optamos por realizar pruebas de susceptibilidad. Existe evidencia en la literatura especializada de que los valores de las CIMs $\leq 1 \mu \mathrm{g} / \mathrm{mL}$, contra la mayoría de los antifúngicos, se relacionan con una buena respuesta terapéutica. Además, el conocimiento del agente etiológico y el perfil de susceptibilidad de los hongos aislados, en pacientes imunocomprometidos, es importante ya que éstos pueden producir infecciones graves con diseminación a otros órganos y sistemas ${ }^{12}$.

La prueba de susceptibilidad fue realizada utilizando la técnica de microdilución en caldo, según el documento M38A del CLSI para hongos filamentosos ${ }^{13}$. Los resultados de susceptibilidad se presentan en la Tabla 1. La cepa de $C$. gloeosporioides presentó CIM bajas para 
anfotericina $\mathrm{B}$ y voriconazol y altas para 5-fluorcitosina y fluconazol. Por otra parte, la cepa de $A$. infectoria presentó resistencia a los cinco antifúngicos utilizados.

\section{Discusión}

Se define como feohifomicosis a un grupo heterogéneo de enfermedades fúngicas caracterizadas por la presencia en los tejidos afectados de hifas, pseudohifas, formas levaduriformes melanizadas o una combinación de estas estructuras $^{14-16}$. Las infecciones producidas por Alternaria han sido descritas en pacientes inmunocomprometidos, aunque también se ha observado en pacientes inmunocompetentes $^{8,17,18}$. Según Pastor y Guarro ${ }^{8}, A$. infectoria es la especie más aislada en clínica y el trasplante es el factor de riesgo más frecuente en los casos de alternariosis cutánea y subcutánea. En general, la presentación clínica de la alternariosis subcutánea es un nódulo en las extremidades, el que puede llegar a ulcerar. También puede presentarse como infiltración ${ }^{18}$, masa vegetativa ${ }^{19} \mathrm{y}$ úlcera ${ }^{20}$. La diseminación sistémica con múltiples lesiones de piel e infiltración pulmonar también ha sido descrita para la especie $A$. infectoria ${ }^{21}$.

El género Colletotrichum incluye varias especies. Es un fitopatógeno clásico, infrecuente en el ser humano. La presentación clínica más común es la keratomicosis y su manifestación subcutánea es rara. Guarro y cols. ${ }^{22}$, comunicaron el primer caso de feohifomicosis causado por $C$. gloeosporioides en un paciente diabético en tratamiento con corticoesteroides. O' Quinn y cols. ${ }^{23}$, describieron tres casos de feohifomicosis subcutánea en pacientes sometidos a quimioterapia por enfermedades hematológicas. Castro y cols. ${ }^{11}$, informaron el quinto caso en un paciente trasplantado renal infectado con $C$. crassipes y recientemente, $C$. gloeosporioides fue descrito en un paciente trasplantado de pulmón ${ }^{17}$.

Nuestro paciente presentó un cuadro clínico causado por dos hongos dematiáceos distintos. Está claro que no fue una co-infección, puesto que existió un período de 12 meses entre la aparición de la primera y segunda infección. Esta situación demostró la importancia de repetir los exámenes de identificación fúngica, aun cuando se sospeche de una recurrencia.

No existe un tratamiento estandarizado para la feohifomicosis pero la cirugía es obligatoria ${ }^{4,24}$. En pacientes inmunocomprometidos se recomienda la combinación de la cirugía y antifúngicos para evitar su diseminación, especialmente en situaciones donde no es factible la resección completa del tejido infectado. Aunque itraconazol puede interactuar con ciclosporina y tacrolimus, fármacos inhibidores de calcineurina utilizados en pacientes trasplantados, es el antifúngico de elección en la feohifomicosis pero la duración del tratamiento y las dosis siguen siendo de carácter empírico ${ }^{25}$. La terbinafina puede ser otra opción y fue utilizada con éxito en un paciente trasplantado con una infección por $A$. tenuissima ${ }^{26}$. Otras alternativas descritas en la literatura científica son voriconazol, posaconazol y caspofungina ${ }^{27}$. Cuando existe infección sistémica, la disminución de la inmunosupresión es necesaria, pero siempre debe asociarse a un antifúngico. Anfotericina B, por su nefrotoxicidad, debe ser utilizada en su formulación liposomal ${ }^{21}$.

Para definir la mejor opción terapéutica siempre debe realizarse una prueba de susceptibilidad. En este caso fueron probados cinco antifúngicos (anfotericina B, itraconazol, 5-fluorcitosina, fluconazol y voriconazol) contra las cepas aisladas. Anfotericina B y voriconazol mostraron CIMs bajas para C. gloeosporioides lo que indica que este hongo fue susceptible a estos antifúngicos. Alternaria infectoria presentó CIMs muy altas para los antifúngicos probados. Resultados similares fueron obtenidos por Badali y cols. ${ }^{28}$, en 70 muestras clínicas y ambientales de Alternaria spp. probadas contra ocho fármacos antifúngicos (anfotericina $\mathrm{B}$, itraconazol, fluconazol, voriconazol, isavuconazol, posaconazol, caspofungina y anidulafungina). Presentaron resistencia a anfotericina B, itraconazol, fluconazol, voriconazol, isavuconazol y caspofungina.

En el caso presentado se optó por no utilizar ningún antifúngico después de la resección total de las lesiones. El paciente fue evaluado durante cinco años, sin presentar ninguna recaída.

Este caso, con infección sucesiva por $A$. infectoria y $C$. gloeosporioides, ilustra el rol de estos hongos ambientales como agentes etiológicos de feohifomicosis en pacientes inmunocomprometidos, así como también demuestra la importancia de investigar y cultivar todas las lesiones sospechosas, para así realizar un diagnóstico correcto para el mejor manejo clínico del paciente.

\section{Resumen}

Diversas especies de hongos negros han sido descritos como agentes de feohifomicosis subcutáneas. A pesar que la gran mayoría de estos se consideran como oportunistas, juegan un papel importante en la feohifomicosis, enfermedad considerada una micosis emergente en pacientes trasplantados de órganos sólidos. Reportamos un caso de feohifomicosis subcutánea causada por Alternaria infectoria en el dorso de la mano izquierda y $4^{\circ}$ falange de la mano derecha de un paciente de sexo masculino de 68 años de edad, que había sido sometido a un trasplante de riñón 35 meses antes. Se realizó escisión quirúrgica de la lesión. Después de un año, el paciente presentó una nueva lesión, esta vez en la $5^{\circ}$ falange de la mano derecha causada por Colletotrichum gloeosporioides, lesión que también fue removida quirúrgicamente. El paciente no 
presentó nuevas lesiones después de las cirugías. Las pruebas de susceptibilidad antifúngica fueron realizados utilizando cinco antifúngicos (anfotericina B, itraconazol, 5-fluorcitosina, fluconazol y voriconazol). Alternaria infectoria presentó resistencia a los cinco antifíngicos y $C$. gloeosporioides fue susceptible solamente a anfotericina B y voriconazol. Enfatizamos la necesidad de realizar estudios histopatológicas y microbiológicos de nuevas lesiones de feohifomicosis, ya que el mismo paciente fue infectado por dos hongos diferentes.

\section{Referencias bibliográficas}

1.- Virgili A, Zampino M, Mantovani L. Fungal skin infections in organ transplant recipients. Am J Clin Dermatol 2002; 3: 19-35.

2.- Revankar S G, Patterson J E, Sutton D A, Pullen R, Rinaldi M G. Disseminated phaeohyphomycosis: review of an emerging mycosis. Clin Infect Dis 2002; 34: 467-76.

3.- Revankar S G. Dematiaceous fungi. Mycoses 2007; 50: 91-101.

4.- Ogawa M M, Galante N Z, Godoy P, Fischman-Gompertz O, Martelli F, Colombo AL, et al. Treatment of subcutaneous phaeohyphomycosis and prospective follow-up of 17 kidney transplant recipients. J Am Acad Dermatol 2009; 61: 977-85.

5.- De Bièvre C. Les Alternaria pathogènes pour l'homme: mycologie épidémiologique. J Mycol Méd 1991; 118: 50.

6.- Farina C, Gotti E, Parma A, Naldi L, Goglio A Pheohyphomycotic soft tissue disease: caused by Alternaria alternata in a kidney transplant patient: a case report and literature review. Transpl Proc 2007; 39: 1655-9.

7.- De Hoog G S J, Guarro J, Gene J, Figueras M J. Atlas of Clinical Fungi, 2nd ed. Centralbureau voor Schimmelcultures, Utrecht, The Netherlands 2000.

8.- Pastor F J, Guarro J. Alternaria infections: laboratory diagnosis and relevant clinical features. Clin Microbiol Infect Dis 2008; 14: 734-46.

9.- Photita W, Photia W, Taylor P W J, Ford R, Hyde K D, Lumyong S. Characterization and pathogenicity of Colletotrichum species associated with anthracnose on chilli (Capsicum spp.) in Thailand. Plant Pathol 2008; 57: 562-72.

10.- Cano J, Guarro J, Gené J. Molecular and morphological identification of Colletotrichum species of clinical interest. J Clin Microbiol 2004; 42: 2450-4.

11.- Castro L G, da Silva Lacaz C, Guarro J, Gené J, Heins-Vaccari E M, de Freitas Leite R S, et al. Phaeohyphomycotic cyst caused by Colletotrichum crassipes. J Clin Microbiol 2001; 39: 2321-4.

12.- Revankar S G, Sutton D A. Melanized fungi in human disease. Clin Microbiol Rev 2010; 23 : 884-928.

13.- National Committee for Clinical Laboratory Standards. Reference method for broth dilution antifungal susceptibility testing of filamentous fungi. Approved standard M38-A. National Committee for Clinical Laboratory Standards, Wayne, PA.

14.- Ajello L, Georg L K, Steigbigel R T, Wang C J. A case of phaeohyphomycosis caused by a new species of Phialophora. Mycologia 1974; 66: 490-8.

15.- McGinnins M R. Chromoblastomycosis and phaeohyphomycosis: new concepts, diagnosis and mycology. J Am Acad Dermatol 1983; 8: 1-16.

16.- Sood N, Gugnani H C, Guarro J, PaliwalJoshi A, Vijayan V K. Subcutaneous phaeohyphomycosis caused by Alternaria alternata in an immunocompetent patient. Int J Dermatol 2007; 46: 412-3.

17.- Severo C B, Oliveira F de M, Pilar E F, Severo L C. Phaeohyphomycosis: a clinicalepidemiological and diagnostic study of eighteen cases in Rio Grande do Sul, Brazil. Mem Inst Oswaldo Cruz 2012; 107: 854-8.

18.- Romano C, Valenti L, Miracco C, Alessandrini C, Paccagnini E, Faggi E, et al. Two cases of cutaneous phaeohyphomycosis by Alternaria alternata and Alternaria tenuissima. Mycopathologia 1997; 137: 65-74.

19.- Romano C, Vanzi L, Massi D, Difonzo E M. Subcutaneous alternariosis. Mycoses 2005; 48 : 408-12.
20.- Acland K M, Hay R J, Groves R. Cutaneous infection with Alternaria alternata complicating immunosuppression: successful treatment with itraconazole. Br J Dermatol 1998; 138: 354-6.

21.- Halaby T, Boots H, Vermeulen A, van der Ven A, Beguin H, Van Hooff H, et al. Phaeohyphomycosis caused by Alternaria infectoria in a renal transplant recipient. J Clin Microbiol 2001; 39: 1952-5.

22.- Guarro J, Svidzinski T E, Zaror L, Forjaz M H, Fishman O. Subcutaneous hyalohyphomycosis caused by Colletotrichum gloeosporiodes. J Clin Microbiol 1998; 36: 3060-5.

23.- O’Quinn R P, Hoffmann J L, Boyd A S. Colletotrichum species as emerging opportunistic fungal pathogens: a report of 3 cases of phaeohyphomycosis and review. J Am Acad Dermatol 2001; 45: 56-61.

24.- Clancy C J, Wingard J R, Hong Nguyen M. Subcutaneous phaeohyphomycosis in transplant recipients: review of the literature and demonstration of in vitro synergy between antifungal agents. Med Mycol 2000; 38: 169-75.

25.- Sharkey P K, Graybill J R, Rinaldi M G, Stevens D A, Tucker R M, Peterie J D, et al. Itraconazole treatment of phaeohyphomycosis. J Am Acad Dermatol 1990; 23: 577-86.

26.- Altomare G F, Capella G L, Boneschi V, Viviani M A. Effectiveness of terbinafine in cutaneous alternariosis. Br J Dermatol 2000; 142: 840-1.

27.- Boyce R D, Deziel P J, Otley C C, Wilhelm M P, Eid A J, Wenngenack N L, et al. Phaeohyphomycosis due to Alternaria species in transplant recipients. Transpl Infect Dis 2010; 12: $242-50$.

28.- Badali H, De Hoog G S, Curfs-Breuker I, Andersen B, Meis J F. In vitro activities of eight antifungal drugs against 70 clinical and environmental isolates of Alternaria species. J Antimicrob Chemother 2009; 63: 1295-7. 